\title{
Quelques exemples d'influence de la géométrie des structures situées à l'aspiration d'une roue de pompe sur son comportement en cavitation
}

\author{
J. F. Lapray, R. Canavelis \\ Bergeron-Rateau
}

L'influence des conditions d'aspiration et d'installation des pompes rotodynamiques sur les courbes caractéristiques autres que le NPSH est un problème qui a fait l'objet de nombreux travaux et recherches essentiellement à caractère expérimental. Les connaissances accumulées et l'expérience industrielle passée permettent aux constructeurs et installateurs, à défaut de maitriser parfaitement la question, d'arriver à des compromis technico-économiques satisfaisants pour le bon comportement hydromécanique des pompes.

Par contre on dispose de beaucoup moins d'éléments quantifiables en ce qui concerne l'influence des conditions d'aspiration des pompes sur leur comportement à la cavitation.

Nous nous proposons, à l'aide de quelques résultats expérimentaux, de montrer comment, pour une même pompe, les courbes de cavitation peuvent être modifiées quand on change la géométrie en amont immédiat d'une roue de pompe.

\section{Généralités}

\subsection{Rappel de notions de base}

Le nombre de cavitation $\sigma_{0}$ relatif à l'apparition de la cavitation sur une entrée d'aube de pompe peut être relié au NPSH par la relation:

$$
(\mathrm{NPSH})_{0}=\frac{\bar{V}_{1}^{2}}{2 g}\left(\sigma_{0}+1\right)+\sigma_{0} \frac{\bar{U}_{1}^{2}}{2 g}
$$

où

$$
\sigma_{0}=\frac{P_{1}-P_{v}}{\bar{W}_{1}^{2} / 2 g} \times F\left(i_{1}\right)
$$

avec

$$
\vec{V}_{1}=\vec{W}_{1}+\vec{U}_{1}
$$

$\vec{V}_{1} \quad=$ Vitesse absolue du fluide à l'entrée pompe.

$\vec{W}_{1} \quad=$ Vitesse relative du fluide à l'entrée d'aube sur un diamètre $D_{1}$ de référence.

$\vec{U}_{1} \quad=$ Vitesse d'entraînement de l'aube au diamètre $D_{1}$.

$P_{1} \quad=$ Pression absolue à l'entrée pompe.

$P_{v} \quad=$ Pression de vapeur saturante du fluide pompé.

$F\left(i_{1}\right)=$ Coefficient fonction de l'incidence $i_{1}$ des filets fluides.

Pour un fluide pompé à température constante et pour une géométrie donnée de l'aube, $\sigma$ ne dépend que de $\vec{W}_{1}$ et $P_{1}$.

Si la pression $P_{1}$ à l'entrée de la roue est maintenue

Influence of the geometry of structures located at a pump suction point on its cavitation behaviour.

In many cases, pumps users start the pumping installation by relying on the contractual characteristic curves supplied by the manufacturer. They also have other curves defining the NPSH in accordance with the flow rate. These curves have been established in extremely special conditions which correspond to the ideal case of a narrow pipeline pump suction with a homogeneous supply and a quasi-uniform approach speed distribution.

With the help of some examples, this article seeks to draw attention to the modifications which could affect these curves when the actual installation differs from this ideal situation. 
constante, $\sigma_{0}$ ne dépend que de $\vec{W}_{1}$.

Mais $\vec{W}_{1}$ ne dépend que de $\vec{V}_{1}$ pour un point fixé de l'aube. On voit que toute variation de la vitesse absolue $\vec{V}_{1}$ en module et en direction à l'entrée de la pompe a une influence sur le nombre de cavitation $\sigma_{0}$ et sur le $(\mathrm{NPSH})_{0}$ correspondant à l'apparition de la cavitation.

On peut définir de la même manière l'influence de $\vec{V}_{1}$ sur les valeurs du NPSH correspondant à $0 \%, 3 \%$ ou $12 \%$ de chute de la hauteur totale d'élévation.

\subsection{Modifications des courbes de NPSH}

Le cas idéal d'alimentation d'une pompe est celui délivré par une tuyauterie droite de longueur suffisante ( 20 ou $30 \mathrm{D})$ et d'un diamètre légèrement supérieur au diamètre d'entrée de l'œillard de la roue d'aspiration de la pompe.

Dans ces conditions le profil des vitesses à l'entrée de la roue est dénué de composante rotationnelle, quasi uniforme, axisymétrique et présente un taux de turbulence faible.

Les entrées d'aubages, au point dit « d'adaptation » de l'œillard de la pompe, sont, en général, étudiées pour un écoulement de ce type.

Dans la pratique, il est rare de pouvoir disposer d'un écoulement de cette qualité, pour des soucis de réduction de génie civil et d'encombrement ; on se trouve donc souvent en présence d'un écoulement avec des écarts par rapport au profil idéal de calcul. La qualité d'une prise d'eau ou d'un organe d'aspiration des pompes peut ainsi

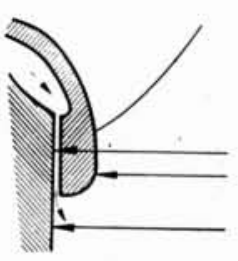

bague $A$

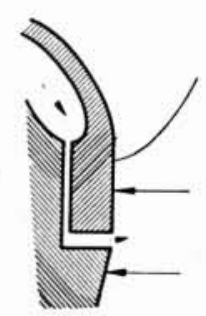

bague $\mathrm{B}$

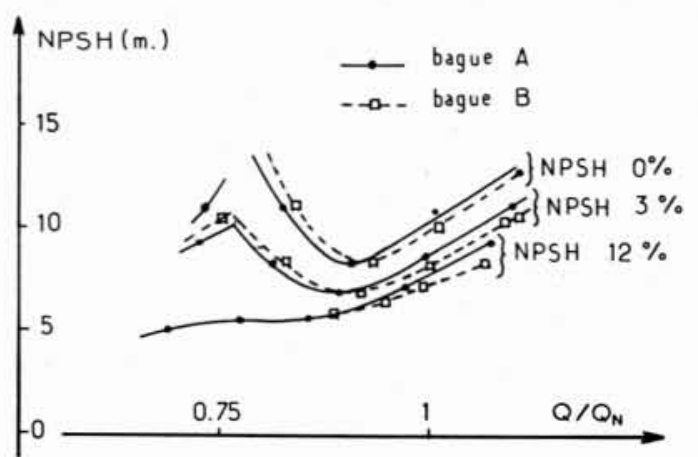

1. Influence de la forme de la bague d'étanchéité du labyrinthe d'aspiration. se mesurer par son degré de « déviation " par rapport à l'écoulement «idéal " décrit précédemment :

- taux de distorsion des vitesses locales par rapport au profil « idéal ",

- composante tangentielle résiduelle du fluide,

- degré de turbulence.

Ces écarts inévitables ont bien évidemment des conséquences sur les courbes de cavitation des pompes.

\subsection{Conditions générales d'essai}

L'ensemble des essais présentés a été réalisé sur la même boucle d'essai, en utilisant des conditions expérimentales répétitives.

Les pompes sont installées sur une boucle en circuit fermé et sont alimentées par un ballon mis en dépressurisation par des pompes à vide. Le fluide d'essai est de l'eau industrielle propre et froide satisfaisant aux caractéristiques physiques recommandées entre autres dans les codes d'essai ISO.

L'incertitude de mesure sur les NPSH et les hauteurs totales d'élévation est inférieure à $0,5 \%$; sur les débits, elle est inférieure à $1 \%$.

Les essais présentés sont relatifs à des pompes dont les diamètres d'entrée sont supérieurs à $200 \mathrm{~mm}$ pour des vitesses de rotation comprises entre 1200 et $1500 \mathrm{tr} / \mathrm{min}$.

\section{Influence de structures internes à la pompe}

\subsection{Bagues d'étanchéité des labyrinthes d'aspiration}

Les labyrinthes d'étanchéité ont pour fonction de limiter le débit de fuite entre le rotor et le stator. Ils peuvent présenter des formes variées liées à un grand nombre de paramètres (taille, matériau, type d'eau véhiculée, etc.).

Le cas expérimental présenté figure 1 est relatif à une pompe de vitesse spécifique 90 ; il compare les résultats obtenus avec deux géométries de bagues d'une roue de diamètre d'entrée $250 \mathrm{~mm}$.

Avec la bague de type $A$, la fuite n'est pas retournée ; l'écoulement principal converge brusquement du diamètre extérieur de la bague au diamètre d'œillard de la roue.

La bague de type $B$, plus classique, dévie le débit de fuite de $90^{\circ}$ et l'écoulement principal converge avant l'entrée de la roue.

Les essais, réalisés avec la même roue, et les mêmes diamètres d'étanchéité et jeux radiaux montrent un léger décalage vers les grands débits des courbes de NPSH de la pompe munie d'une bague de type $B$. A débit partiel, vers le débit de recirculation d'œillard, les deux bagues n'apportent pas de modifications notables.

On peut penser que c'est la différence de gradient de vitesse dans la couche limite à l'entrée de la roue qui influe sur le comportement en cavitation de l'aubage à proximité de la ceinture. Une visualisation stroboscopique de la roue de type $A$, en surdébit, montre l'apparition de figures de cavitation sur l'arrondi du flasque en entrée de roue qui n'apparaît pas dans le cas de la pompe munie d'une bague de type $B$. 


\subsection{Influence d'un croisillon et d'une ogive fixe à l'entrée d'une pompe hélice avec coude d'aspiration}

Ces essais ont été effectués sur une pompe hélice de vitesse spécifique 220 de diamètre $350 \mathrm{~mm}$ alimentée par un coude convergent.

Dans la plupart des cas la pointe de roue ou ogive est solidaire du rotor.

Cependant pour des raisons constructives il est parfois utile de pouvoir disposer un palier en tête de pompe. Ce dernier est en principe installé dans l'ogive qui est solidaire du corps de pompe par l'intermédiaire de « bras profilés » qui jouent alors le rôle de croisillon.

La figure 2 présente les essais de cavitation d'une pompe hélice essayée dans les deux configurations décrites ci-dessus.

On constate, principalement sur les courbes de (NPSH)-3\% et (NPSH)-12\% une amélioration en surdébit des performances en cavitation due à la présence du croisillon formé par les 4 bras supportant l'ogive fixe.

On peut penser que ce croisillon et l'absence de rotation de l'ogive contribuent au bon redressement des vitesses de sortie du coude dans le sens axial (absence de composante tangentielle).

En revanche les courbes de cavitation de l'hélice avec ogive fixe présentent des irrégularités qui confirment que, sur les pompes hélices, le comportement des aubages est très sensible à des petites modifications de l'écoulement d'entrée telles que les sillages des croisillons.

\subsection{Influence des trous d'équilibrage}

Les essais décrits figure 3 concernent une pompe centrifuge de vitesse spécifique 62 avec un diamètre d'entrée de $250 \mathrm{~mm}$.

La pompe est munie d'une bague dite d'équilibrage en son dos. Les essais ont consisté, entre autre, à effectuer les essais d'aspiration dans deux configurations ;

- Débit de fuite à travers la bague d'étanchéité évacué vers l'extérieur par une tuyauterie d'équilibrage placée sur le fond de corps de pompe.

- Retour du débit de fuite à l'entrée pompe à l'aide de 6 trous d'équilibrage percés dans le flasque arrière de la roue.

Les essais montrent, de façon très nette, une altération de la courbe de (NPSH)-3\% de la pompe dont le débit de fuite retourne dans l'entrée roue à travers les trous d'équilibrage. Cela se comprend aisément du fait que le débit de fuite qui représente 1 ou $2 \%$ du débit principal perturbe, l'écoulement principal le long du flasque arrière de la roue.

A débit partiel, vers la zone du débit de recirculation de l'entrée roue, l'influence est plus grande et la courbe de (NPSH)-3\% de la roue avec trous d'équilibrage possède une remontée caractéristique plus nette que dans le cas où il n'y a pas de trous. Ceci tendrait à prouver que le débit de fuite, même faible a un rôle "perturbateur " important quand les écoulements de recirculation à l'entrée roue s'établissent progressivement.

Des études fines expérimentales pourront peut-être, dans le futur, apporter quelques éclaircissements sur cette question.

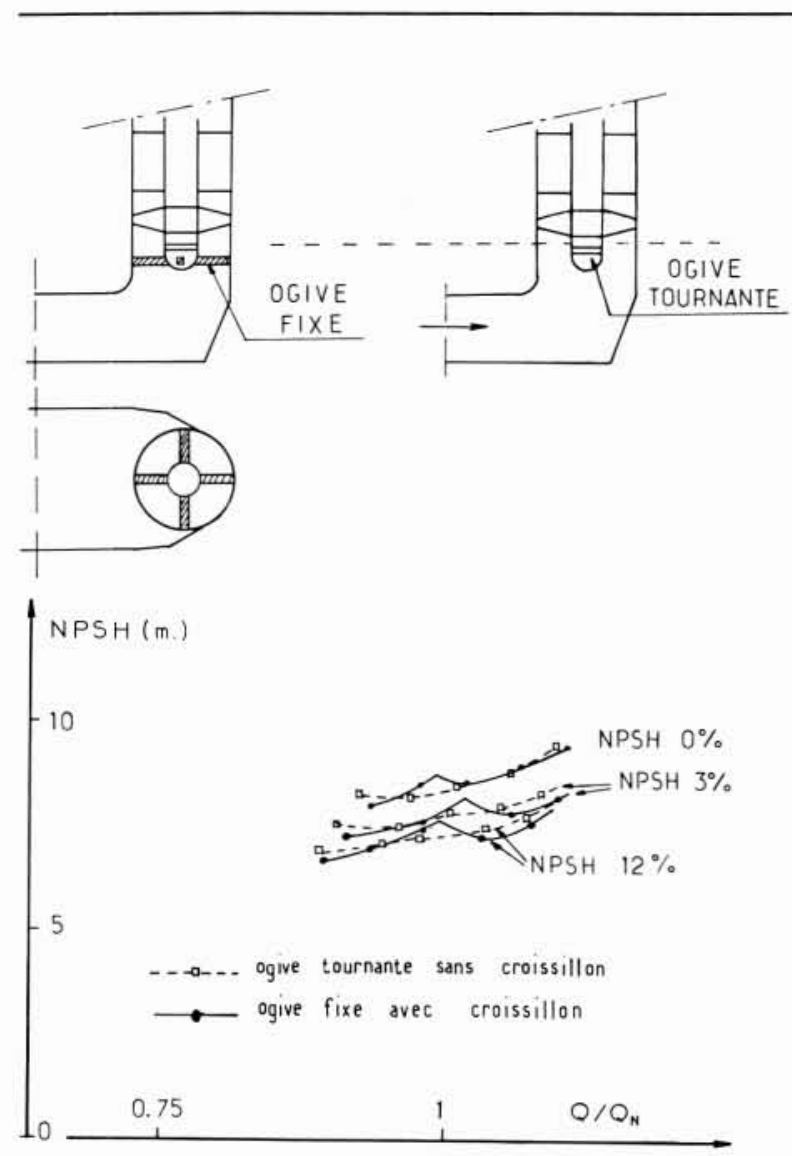

2. Influence d'une ogive fixe et d'un croisillon devant une roue hélice avec coude d'aspiration.
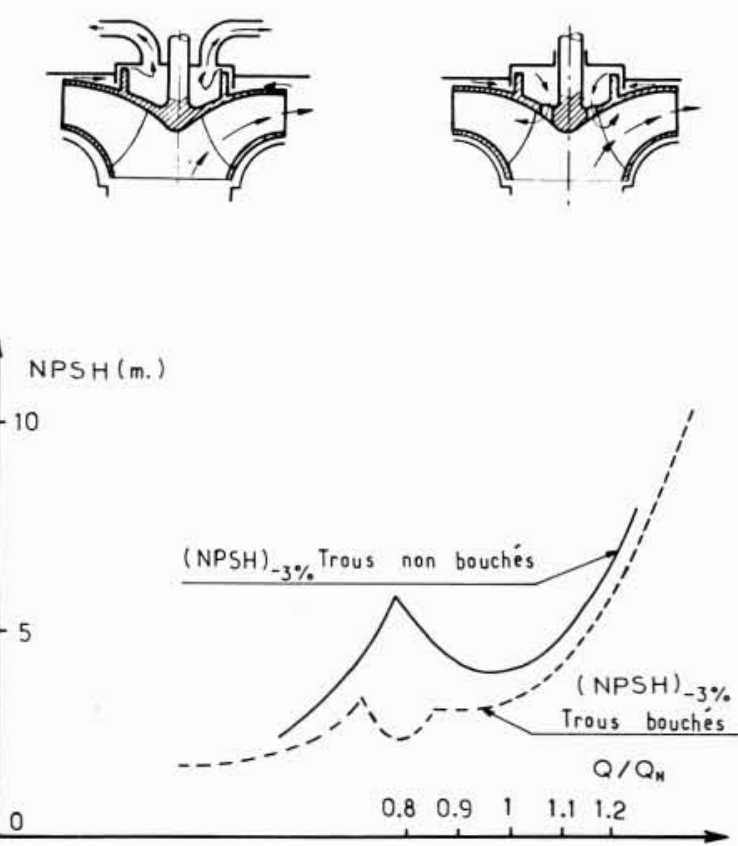

3. Influence des trous d'équilibrage sur les courbes de (NPSH)$3 \%$ 


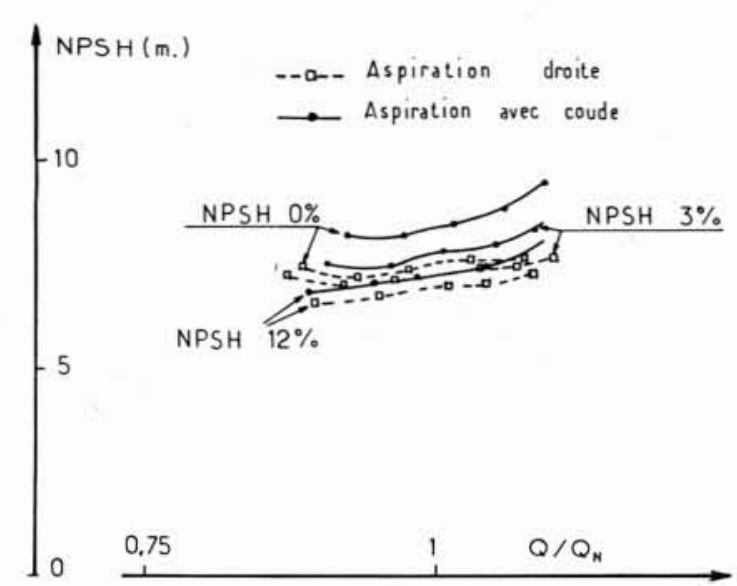

4. Influence d'un coude à l'aspiration d'une pompe hélice.

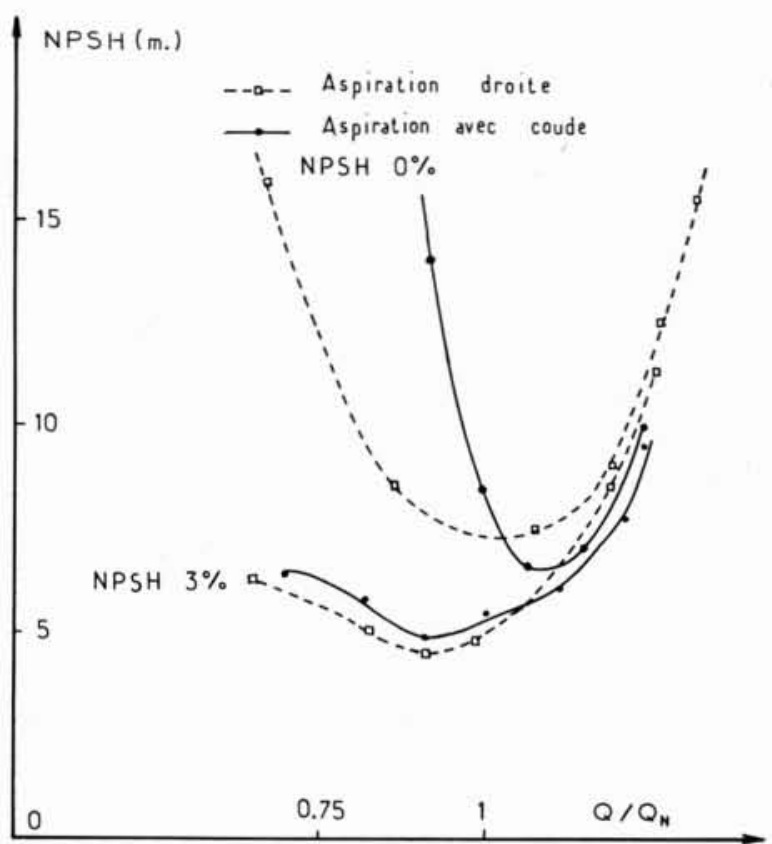

5. Influence d'un coude à l'aspiration d'une pompe centrifuge.

\section{Influence des structures externes}

\subsection{Coudes d'aspiration}

Les conditions réelles d'installation des pompes sur site industriel conduisent fréquemment à l'utilisation de coudes d'aspiration pour alimenter les pompes.

Ces coudes sont souvent convergents entre leur section d'entrée et l'œillard de la pompe, de façon à délivrer un écoulement satisfaisant au mieux aux critères développés au $\$ 1.2$.

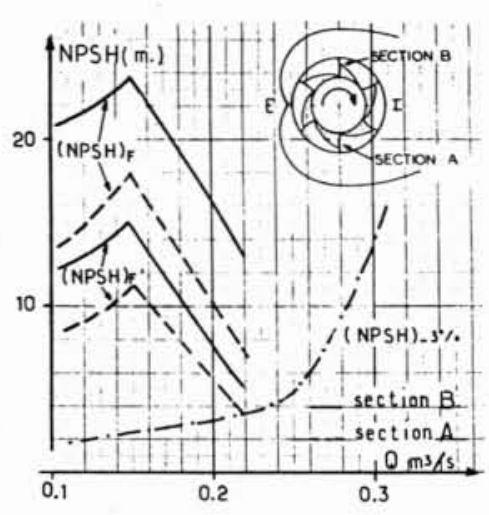

Evolution du NPSH dans deux sections de l'oeillard

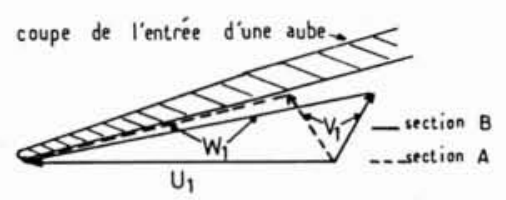

Triangles des vitesses a l'entrée : $0=0.2 \mathrm{m3} / \mathrm{s}$

6.

La figure 4 montre l'influence d'un tel coude sur les courbes de (NPSH) de la pompe hélice déjà étudiée au $\$ 2.2$.

Les courbes présentées permettent de comparer les courbes de cavitation de l'hélice avec ogive tournante montée successivement avec une tuyauterie droite à son aspiration puis avec le coude convergent.

D'une façon générale, le coude a altéré très sensiblement les courbes de cavitation de la pompe hélice qui conduisent, sur toute la plage étudiée, à un NPSH supérieur à celui obtenu avec l'hélice alimentée avec la tuyauterie droite.

La figure 5 montre également la comparaison des ourbes de cavitation d'une pompe centrifuge de ns 90 alimentée successivement en tuyauterie droite et par un coude convergent (de tracé différent du précédent).

On note deux tendances distinctes:

— en surdébit $Q>Q_{N}$, le coude convergent améliore légèrement les courbes de cavitation,

- pour les débits inférieurs au débit nominal on constate une inversion de tendance, avec une forte dégradation des courbes de (NPSH) $0 \%$. Le coude a un effet perturbateur dans le cas considéré sur le fonctionnement à débit partiel de la pompe.

Dans les deux cas présentés, le comportement des roues alimentées par un coude se trouve influencé par les défauts d'écoulement apportés par la présence de ce coude: non-uniformité parfaite des vitesses axiales, composante tangentielle résiduelle, taux de turbulence élevé dans la zone de plus grande courbure du coude. 


\subsection{Culotte d'aspiration}

De construction, les pompes monoétagées à double entrée comportent une culotte d'aspiration formée de deux coudes accolés qui alimentent chacun une des demi-roues. Dans ce cas les courbes de cavitation obtenues aux essais intègrent nécessairement l'influence des coudes.

La figure 6 montre les courbes de cavitation obtenues par visualisation stroboscopique d'une demi-roue d'une pompe à double entrée.

La courbe de (NPSH) $f$ correspond à l'apparition des premières bulles visibles de cavitation sur la face en dépression de l'aube.

La courbe (NPSH) $f^{\prime}$ est relative à un développement des figures devenues instables avec lâchés de poches de vapeur dans l'écoulement.

Les résultats expérimentaux, pour les débits $Q<Q N$ montrent que les courbes de (NPSH) obtenues par visualisation sont variables en fonction de l'azimuth du lieu de l'observation. Comme l'indique la figure 6 ceci est le résultat d'une non-axisymétrie du champ des vitesses absolues délivré par la culotte. Dans le cas présent, un relevé précis de la carte des vitesses en sortie des coudes a permis d'expliquer les écarts trouvés sur le développement des figures de cavitation.

\section{Conclusion}

Les exemples présentés plus haut confirment que le NPSH requis des pompes dépend de façon essentielle de la structure de l'écoulement dans l'œillard de la roue aspiratrice. Il en résulte que le NPSH est très sensible à toute modification de la géométrie des structures situées à l'amont immédiat de cette roue.

En conséquence la détermination précise des courbes prévisionnelles de NPSH nécessite de prendre en compte les conditions réelles d'installation en milieu industriel.

A ce titre une collaboration étroite entre l'installateur et le constructeur s'avère souvent indispensable.

En principe, le constructeur doit pouvoir accéder à la connaissance des écoulements délivrés par les structures de l'installation à la pompe. Cette exigence peut notamment nécessiter des études particulières sur maquette en soufflerie ou en boucle d'essai en eau.

Adresse des auteurs:

Messieurs J. F. Lapray

Bergeron-Rateau

$R$. Canavelis

Le Niemeyer

Avenue des Olympiades

94120 Fontenay-sous-Bois

Tél. : 43948712 
M. AMBLARD: Je voudrais poser deux questions. La première est la suivante: Quelle est l'influence de la forme de la bague d'étanchéité du labyrinthe d'aspiration sur la courbe hauteur-débit de la pompe. En modifiant la réinjection du débit de fuite dans l'aspiration, cela joue sur la recirculation de l'écoulement à l'entrée de la pompe.

A l'époque de la recherche du tracé des turbines-pompes de Revin, avec M. Gurron, nous avons fait des essais avec un labyrinthe du type B pour agir sur la hauteur du « feston » de la courbe hauteur-débit. C'est me semble-t-il ce qui a été réalisé industriellement.

M. LAPRAY : A notre grand étonnement, on a eu en dessous de $0,8 \mathrm{Qn}$, une modification assez sensible de la courbe de puissancedébit et un peu de la courbe de hauteur-débit. Par contre, audessus de $0,8 \mathrm{Qn}$, les écarts entre les courbes puissance-débit et hauteur-débit se confondaient.

M. AMBLARD : Ma seconde question est plutôt un commentaire : vous avez dit que la présence d'un coude à l'aspiration conduisait à une altération des caractéristiques de cavitation par rapport à une tuyauterie droite. Je voudrais ajouter que la présence d'un coude d'aspiration avec arbre traversant entraîne une dyssimétrie de l'écoulement au bord d'attaque des aubes de la roue. Il en résulte que les figures de cavitation sont différentes selon l'azimut des aubes. A ma connaissance, on n'a pas encore réalisé un coude industriel avec arbre traversant permettant une axysimétrie de l'écoulement car le problème est très difficile à résoudre.

M. LAPRAY: C'est la grosse difficulté. Il y a une quinzaine d'années, les gens faisaient cette constatation. Ensuite, on a fait des essais et on pourra bientôt faire des calculs. Pour l'instant, la seule façon sérieuse d'agir est d'abord de dessiner une culotte d'aspiration qui soit de dimension raisonnable; ensuite, il faut l'essayer à l'air ou à l'eau et la modifier. Mais cela ne suffit pas, il faut encore regarder comment la roue qui est montée derrière la culotte va répondre. C'est le genre de travail que je vous ai montré

M. GRISON : Sur les courbes, vous avez présenté des variations de NPSH en fonction d'une section d'aspiration avec un coude ou sans coude. Est-ce que l'on connaît à peu près les variations de vitesses qu'il y avait à la sortie du coude? Vous avez montré que le NPSH variait de $10 \%$. Est-ce qu'on sait quel était le gradient de vitesses?

M. LAPRAY: Des essais ont dû être faits sur ce coude. Je pense que le gradient de vitesses n'est pas mauvais en lui-même parce qu'il a peu d'influence sur les courbes caractéristiques à débit normal. Mais je n'ai pas sous la main les informations à ce sujet, je pourrai vous les procurer.

M. GRISON: C'est un paramètre très important. Il avait été souligné aussi sur le problème d'érosion. On a indiqué que le coude pouvait provoquer des érosions à l'entrée. Avec un bon dessin, on pourrait arranger considérablement les choses.

M. LAPRAY: Je pense que le coude de la seconde hélice, après essais en soufflerie, a des taux de distorsion faibles, de 3 ou $4 \%$ au maximum. Ce sont des sondages qui ont été réalisés à l'aide du tube de Pitot. Avec des moyens d'essais plus performants, notamment avec le vélocimètre à laser, on aurait peut-être des surprises.

M. MARTIN : A propos de la figure 1, pourriez-vous nous dire l'influence du changement de bague d'aspiration sur le NPSH ?

M. LAPRAY : Nous l'avons étudié, mais je n'ai pas les résultats exacts ici. Sur les aubes, il n'y avait rien de bien inquiétant. Par contre, je vous ai dit, au cours de l'exposé, qu'avec la bague sur laquelle l'écoulement convergeait, on a vu en surdébit une cavitation au droit de l'arrondi ; ce qui signifie qu'il y a un décollement de l'écoulement dans cette zone et qu'il provoque une légère obstruction supplémentaire à l'entrée de la roue.

Je peux vous dire qu'on a adopté ce type de bague sur le plan industriel et qu'apparemment le comportement est tout à fait satisfaisant. Ces essais ont été réalisés il y a trois ou quatre ans et des expériences industrielles ont été faites sur trois ou quatre machines équipées de bagues de ce type. Ce type d'essai est intéressant parce qu'il permet, notamment dans les pompes de circulation ou certains types de pompes assez grosses, de réduire les coûts de fabrication et de faire des économies au niveau de la pompe. Ce sont les mécaniciens qui nous ont poussés à étudier ce type de bague ; cela diminue les usinages et la bague est plus facile à monter. Cela rejoint la question qui a été posée tout à l'heure. C'est uniquement ce genre d'économie qui provoque ces essais.

M. DESCLAUX: Je voudrais revenir sur une question concernant l'effet de l'aspiration droite et l'effet de l'aspiration avec coude. Le NPSH global est dégradé de l'ordre de 15 à $20 \%$, le point lui-même est déplacé et on observe une amélioration en surdébit alors qu'il y a détérioration en sous-débit.

M. LAPRAY: Il y a des composantes rotationnelles tangentielles résiduelles à la sortie du coude que l'on peut difficilement éliminer, sauf si on dessine un coude extrêmement encombrant et ce qui n'est pas compatible généralement avec les encombrements des stations de pompage dans lesquelles on installe les machines. Imaginez ce genre de coude avec une hauteur une fois et demie ou deux fois le diamètre d'entrée de la pompe; si le diamètre est de l'ordre de $2 \mathrm{~m}$, cela fait 3 ou $4 \mathrm{~m}$. C'est ce qui fait que la hauteur du coude est liée à toute l'économie du projet. Vous avez pu remarquer sur la figure que le coude alimentant cette pompe hélice est très réduit en hauteur, ce qui fait tout son intérêt. C'est ce qui nous a poussés à l'essayer avec une pompe hélice.

M. DESCLAUX: Les essais ont-ils été faits sur machine réelle ou sur modèle?

M. LAPRAY: Ils ont été faits sur modèle. On a fait des essais en soufflerie sur une petite maquette en polystyrène. Pour la pompe hélice, on a réalisé un coude en fonte avec un excellent respect de la géométrie. Il ne faut négliger aucun détail.

M. CANAVELIS: Je vois que vous êtes un peu étonné par l'influence que peut avoir un coude. Je crois que, dans la plupart des tracés de coude que l'on cherche à soigner particulièrement, on observe deux ou trois phénomènes physiques génants, mais quasiment inévitables, à moins d'avoir des encombrements tels qu'ils deviennent tout à fait incompatibles avec un coût d'installation acceptable.

En général, il y a à la sortie du coude, une non-uniformité résiduelle de la vitesse débitante : il y a toujours quelques pourcents d'écart entre la vitesse au centre et celle aux parois. Un très bon coude peut conduire à un écart résiduel de vitesse axiale de 1 à $3 \%$. Quand on refait les triangles de vitesse à l'entrée des aubages et que l'on compose la vitesse absolue avec la vitesse tangentielle d'entraînement, on constate des petits écarts d'incidence sur la vitesse relative par rapport aux aubages.

Ensuite, en plus de ce phénomène, on n'arrive pas forcément à redresser complètement tous les filets fluides de $90^{\circ}$, ce qui fait qu'il y a également une composante tangentielle résiduelle par rapport à la direction axiale ; cette composante provoque également de petits écarts angulaires de vitesse relative à l'entrée des aubages. C'est notamment le cas de la double roue avec culotte que montrait M. LAPRAY; dans un tel cas, on constate des écarts angulaires de signes différents selon la moitié considérée dans la surface annulaire correspondant à l'entrée de la route. Ces incidences alternées des filets fluides dans le mouvement relatif par rapport aux aubes donnent des cavitations non stationnaires sur l'entrée des aubes.

En outre, ces coudes ont souvent un troisième effet en raison du gradient de pression entre courbure interne et courbure externe : ils induisent des torches internes. Ces écoulements secondaires sont matérialisés souvent par deux tourbillons qui, si la pompe fonctionne à faible NPSH, sont le siège de cavitations bruyantes.

Quand on trace un très bon coude et que l'on a la possibilité de soigner la convergence, on arrive en général à une bonne uniformité des vitesses et à un bon redressement des filets fluides, mais il reste toujours difficile de supprimer ces petites torches.

Ceci explique que l'écoulement à l'entrée de la roue n'est jamais conforme à l'idéal. 